\title{
An Improved Movable 3 photomultiplier (3PM)- $\gamma$ Coincidence Counter Using Logical Sum of Double Coincidences in $\beta$-Channel for Activity Standardization
}

\author{
Han Yull Hwang', Jong Man Lee ${ }^{2}$ \\ ${ }^{1}$ Department of Fire Protection \& Safety Management, Mokwon University, Daejeon, Korea; ${ }^{2}$ Center for Ionizing Radiation, Korea Research Institute of \\ Standards and Science, Daejeon, Korea
}

\section{Original Research}

Received January 8, 2020

Revision February 29, 2020

Accepted April 16, 2020

Corresponding author: Han Yull Hwang

Department of Fire Protection \& Safety Management, Mokwon University, 88 Doanbuk-ro, Seo-gu, Daejeon 35349, Korea

E-mail: hyhwang@mokwon.ac.kr

\begin{abstract}
This is an open-access article distributed under the terms of the Creative Commons Attribution License (http://creativecommons.org/licenses/by-nc/4.0) which permits unrestricted use, distribution, and reproduction in any medium, provided the original work is properly cited.
\end{abstract}

Copyright $\odot 2020$ The Korean Association for Radiation Protection

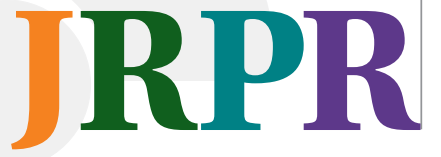

Background: To improve the measurement accuracy of liquid-scintillation counting for activity standardization, it is necessary to significantly reduce the background caused by thermal noise or after-pulses. We have therefore improved a movable 3 photomultiplier (3PM) $-\gamma$ coincidencecounting method using the logical sum of three double coincidences for $\beta$ events.

Materials and Methods: We designed a new data-acquisition system in which $\beta$ events are obtained by counting the logical sum of three double coincidences. The change in $\beta$-detection efficiency can be derived by moving three photomultiplier tubes sequentially from the liquid-scintillation vial. The validity of the method was investigated by activity measurement of ${ }^{134} \mathrm{Cs}$ calibrated at the Korea Research Institute of Standards and Science (KRISS) with $4 \pi(\mathrm{PC}) \beta-\gamma(\mathrm{NaI}(\mathrm{Tl}))$ coincidence counting using a proportional counter (PC) for the $\beta$ detector.

Results and Discussion: Measurements were taken over 14 counting intervals for each liquidscintillation sample by displacing three photomultiplier tubes up to $45 \mathrm{~mm}$ from the sample. The dead time in each $\beta$ - and $\gamma$-counting channel was adjusted to be a non-extending type of $20 \mu$ s. The background ranged about 1.2-3.3 $\mathrm{s}^{-1}$, such that the contributions of thermal noise or afterpulses were negligible. As the $\beta$-detection unit was moved away from the sample, the $\beta$-detection efficiencies varied between 0.54 and 0.81 . The result obtained by the method at the reference date was $396.3 \pm 1.7 \mathrm{kBq} / \mathrm{g}$. This is consistent with the KRISS-certified value of $396.0 \pm 2.0 \mathrm{kBq} / \mathrm{g}$ within the uncertainty range.

Conclusion: The movable 3PM- $\gamma$ method developed in the present work not only succeeded in reducing background counts to negligible levels but enabled $\beta$-detection efficiency to be varied by a geometrical method to apply the efficiency extrapolation method. Compared with our earlier work shown in the study of Hwang et al. [2], the measurement accuracy has much improved. Consequently, the method developed in this study is an improved method suitable for activity standardization of $\beta-\gamma$ emitters.

Keywords: Liquid Scintillation Counting, Movable 3PM- $\gamma$ Coincidence Counter, Logical Sum of Double Coincidences, $4 \pi(\mathrm{LS}) \beta-\gamma(\mathrm{NaI}(\mathrm{Tl}))$ Coincidence Counting, $\beta-\gamma$ Emitter ${ }^{134} \mathrm{Cs}$

\section{Introduction}

We have been successfully developing a movable 3 photomultiplier (3PM)- $\gamma$ device, based on $4 \pi$ (liquid scintillator $[\mathrm{LS}]) \beta-\gamma(\mathrm{NaI}(\mathrm{Tl}))$ coincidence counting method, for activity standardization of pure $\beta$ - or $\beta$ - $\gamma$ emitters $[1,2]$. The three PM tubes placed on the plane are movable from a liquid scintillation vial at the same time, thus enabling the 
variation of $\beta$-detection efficiencies by a geometrical technique to apply the efficiency-extrapolation method. The results obtained with the method were consistent with the ones determined by conventional liquid scintillation counting or $4 \pi$ (proportional counter $[\mathrm{PC}]) \beta-\gamma(\mathrm{NaI}(\mathrm{Tl}))$ coincidence counting. However, for the case of $\beta-\gamma$ emitters, $\beta$-event was determined from linear sum of dynode output signal of each PM tube in our earlier work (see Fig. 2 in reference [2]), thus the background caused by thermal noises or after-pulse could be counted. It was a major factor to overcome in improving measurement accuracy according to our previous work.

Recently, the triple to double coincidence ratio (TDCR) method based on a three PM detection system for liquid scintillation counting [3-5] has been extended to $4 \pi(L S) \beta-\gamma$ coincidence counting [6-8]. Since the thermal noise and the after-pulses detected in each PM tube are in uncorrelated relation each other, thus expected to remove them from the coincidence channel. With this fact, we have planned to improve a movable 3PM- $\gamma$ coincidence counting device by applying TDCR technique, namely the $\beta$-event is determined from the logical sum of three double coincidences. Thus, it is expected to reject thermal noises or after-pulses by the coincidence channels.

The objectives of this study are to show that the method developed through this work can be of an alternative technique suitable for activity standardization of $\beta-\gamma$ emitters. For this, it should investigate the stability of the method against the change of measurement conditions arising from the duration of dead times or thermal noises or after-pulses. So the activity-fluctuations due to above mentioned factors were carefully investigated by varying the duration of dead-times from $5 \mu$ s to $40 \mu$ s. The validity of the method has been demonstrated through the activity standardization of ${ }^{134} \mathrm{Cs}$ calibrated at the Korea Research Institute of Standards and Science (KRISS) by $4 \pi(\mathrm{PC}) \beta-\gamma$ coincidence method using proportional counter for $\beta$-detector.

The design of the movable 3PM- $\gamma$ apparatus, logic signal analyzer and data acquisition device developed for this work are in the following section.

\section{Materials and Methods}

\section{Movable 3PM- $\gamma$ Apparatus Installed at Mokwon University}

The detection unit of 3PM- $\gamma$ coincidence system installed

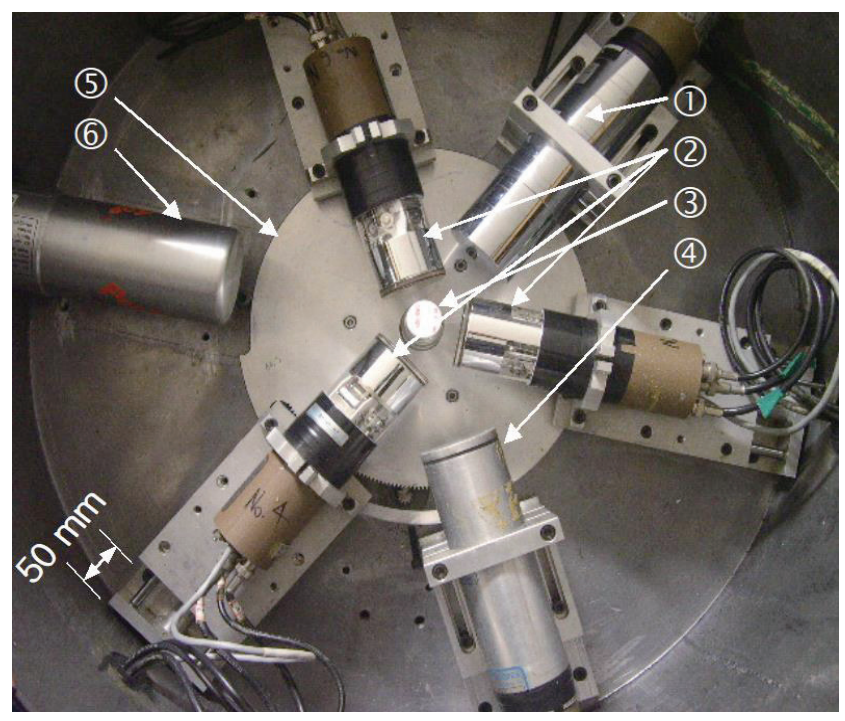

Fig. 1. Photograph of the detection system installed at Mokwon University: (1) $\mathrm{Nal}(\mathrm{TI}) \gamma$-ray detector, (2) three photomultiplier tubes, (3) sample vial, (4) thin window Nal(TI) scintillation detector, (5) platform, (6) HPGe detector. Three photomultiplier tubes can be displaced up to $50 \mathrm{~mm}$ from the centrally located sample vial. HPGe and thin window $\mathrm{Nal}(\mathrm{TI})$ detectors are also placed on the platform to be used for $4 \pi(L S) \beta-\gamma(\mathrm{HPGe})$ coincidence and $4 \pi(\mathrm{LS}) \beta-X$ coincidence counting. Reprinted from Hwang et al. [2] with permission from Elsevier.

at Mokwon University is shown in Fig. 1. Three photo-detectors used for $\beta$-detectors are the PM tubes of Hamamatsu R1847-07 with bi-alkali photocathode of $51 \mathrm{~mm}$ in diameter. These $\beta$-detectors in the plane were designed to move back and forth from centrally located liquid scintillation vial by a connecting gear. A vise ties up them to move at a same time manually up to $50 \mathrm{~mm}$ by rotating a saw-toothed gear. Another three photon detectors, NaI(Tl), HPGe, and X-ray detector, are located $120 \mathrm{~mm}$ rear from the front of $\beta$-detectors not to screen the scintillation pulses incident on the windows. In the present work, a NaI(Tl) detector, $254 \mathrm{~mm}$ (long) $\times 251$ $\mathrm{mm}$ (diameter) was used in the $\gamma$-counting channel. Detection unit is located in a cylindrical chamber of $650 \mathrm{~mm}$ in inner diameter and $320 \mathrm{~mm}$ high and the outer wall is shielded by a $20-\mathrm{mm}$ thick lead plate.

\section{Data Acquisition Method}

The dynode outputs of each PM tube in the $\beta$-channel is amplified and converted to a positive transistor-transistor logic (TTL) signal with $500 \mathrm{~ns}$ width at single channel analyzer (SCA). Thermal noises could be rejected at coincidence channels, so the threshold level of each $\gamma$-counting channel was lowest level $+60 \mathrm{mV}$ of applied SCA. Three double coin- 


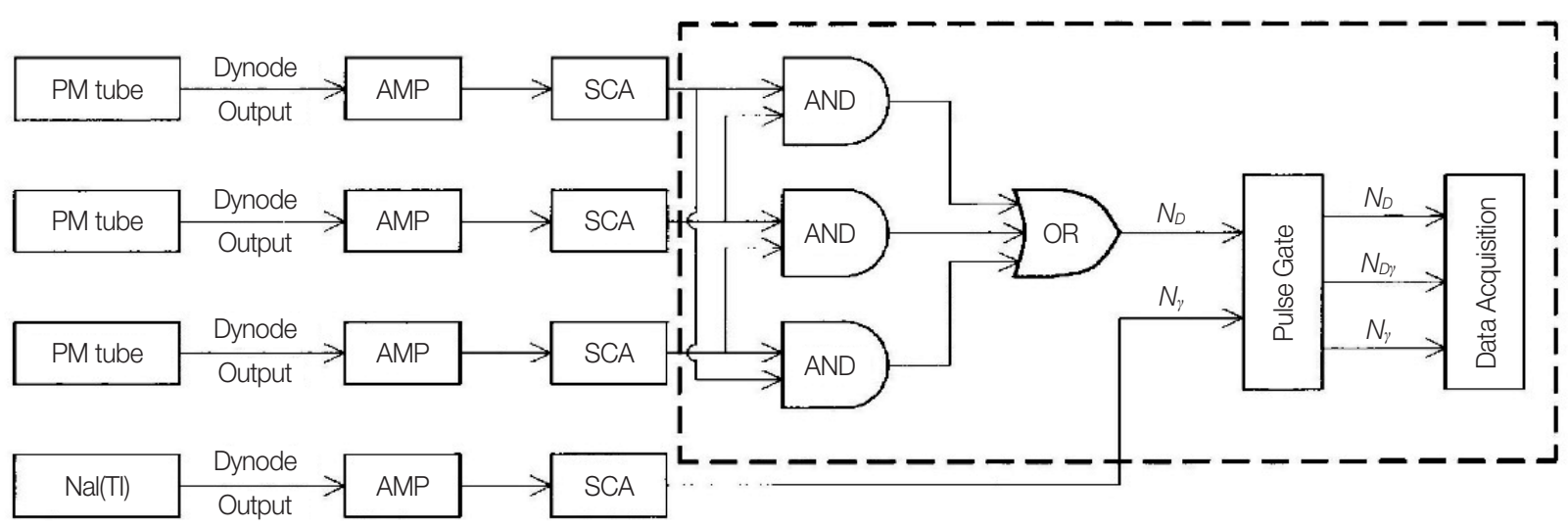

Fig. 2. Simplified block diagram of the applied electronics. The modules being inside rectangular box are homemade logic signal analyzers for this work. PM, photomultiplier; AMP, amplifier; SCA, single channel analyzer.

cident events from these signals are analyzed and their logical sum $N_{D}$ is obtained ( $N_{D}$ becomes $\beta$-events). The resolving time was $500 \mathrm{~ns}$, the width of the SCA output signal. Both the SCA output $N_{\gamma}$ from $\gamma$-channel and the $N_{D}$ are input to Pulse Gate, custom-made at the factory for this work. It was fabricated in an integrated circuit so that it not only adjusts the delay time and pulse width of each output pulse, but also has the ability to do AND/OR works. All operations are controlled by the RSC 232 serial communication port. Delay time and pulse width, whichever is longer, becomes the dead time of corresponding counting channel. It is non-extending type. Outputs from Pulse Gate, $N_{D}, N_{\gamma}$ and coincidence counting $N_{D y}$, are recorded in Data Acquisition device. The simplified block diagram of the applied electronics is displayed Fig. 2. The modules laying inside rectangular box are logic signal analyzers specially designed for this work.

\section{Sample Preparation}

Liquid scintillation samples were prepared by mixing aliquots of a radioactive solution of ${ }^{134} \mathrm{Cs}$ with $10 \mathrm{~mL}$ liquid scintillator. It was same one used at our earlier work [2]. This radionuclide is very important for calibration of $\gamma$-ray spectrometry and also used as a tracer for ${ }^{137} \mathrm{Cs}$ activity determination $[9,10]$. It decays mainly by $\beta$-emission to ${ }^{134} \mathrm{Ba}$. In the de-excitation process, $11 \gamma$-emissions are identified [11]. The coincidence window was set on $796 \mathrm{keV}$ full-energy peak. The liquid scintillation samples were prepared by filling 20 $\mathrm{mL}$ standard glass vials with $10 \mathrm{~mL}$ of unquenched Ultima Gold liquid scintillator (PerkinElmer, Waltham, MA, USA). The observed counting rates were from $3 \times 10^{3} \mathrm{~s}^{-1}$ to $6 \times 10^{3}$ $\mathrm{s}^{-1}$. The measurement was carried out for 14 counting intervals for each prepared sample by displacing three PM tubes up to $45 \mathrm{~mm}$ from a counting sample, up to $30 \mathrm{~mm}$ in steps of $3 \mathrm{~mm}$ and then to $45 \mathrm{~mm}$ in steps of $5 \mathrm{~mm}$. Backgrounds were measured likewise.

\section{Results and Discussion}

\section{Validation of Geometrial Efficiency Variation Method}

Fig. 3 shows logical sums of three double coincidences rate $\left(N_{D}\right)$ observed at each counting interval with a sample. As the $\beta$-detection unit, three PM tubes, was moving away from a centrally located counting vial, it was decreasing absolutely up to $30 \%$ according to our data, thus expecting to obtain the efficiency function defined on a sufficient region to extrapolate linearly. Inset shows observed background versus displacement. It was about $3.3 \mathrm{~s}^{-1}$ to $1.2 \mathrm{~s}^{-1}$ for every cases of measurement. For the case of a sample of count rate of 3,000 s-1, effects of background on observed activity is about $0.08 \%$ according to data. It was $0.57 \%$ with our earlier method (Table 1), so it was a major factor in reducing measurement accuracy. However, we could confirm the backgrounds due to thermal noises or after-pulses were almost rejected at coincidence channel to negligible levels.

\section{Linear Extrapolation of Efficiency Function}

The dead time of each $\beta$ - and $\gamma$-counting channel was adjusted to be non-extending type of $20 \mu \mathrm{s}$ (see next paragraph). The true coincidence rate $\rho_{\beta}$, true $\beta$ count rate $\rho_{\beta}\left(=N_{D}\right)$, true $\gamma$ count rate $\rho_{\gamma}$ and $\beta$-detection efficiency $\varepsilon_{\beta}\left(=\rho_{\beta \gamma} / \rho_{\gamma}\right)$ were corrected and determined by using the mathematical formula derived by Cox and Isham [12], and completed by Smith [13].

Fig. 4 shows a plot of an efficiency function obtained at each counting interval. The parameter of $\mathrm{x}$-axis is $\left(1-\varepsilon_{\beta}\right) / \varepsilon_{\beta}$ 


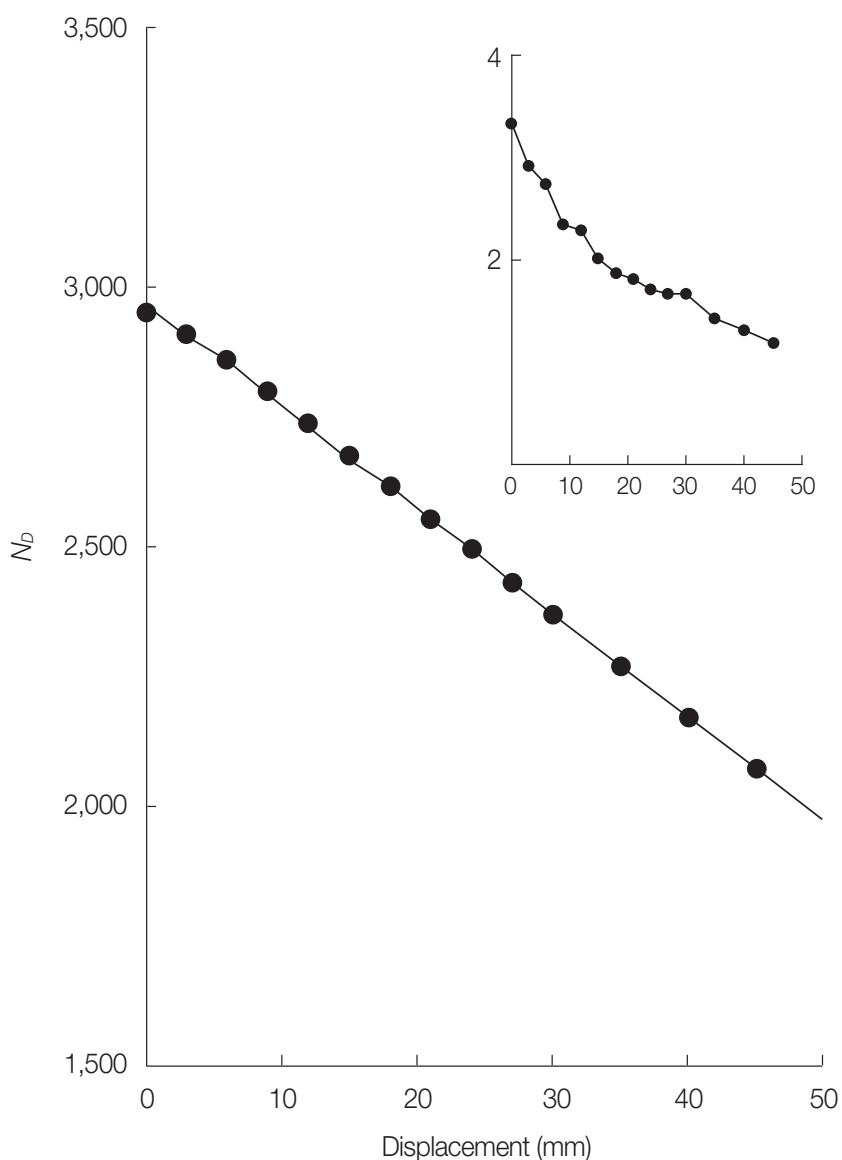

Fig. 3. Logical sum of double coincidence rate, $N_{D}$, observed at each counting interval. The measurement was carried out for 14 counting intervals by displacing three PM tubes up to $45 \mathrm{~mm}$ from a liquid scintillation sample, up to $30 \mathrm{~mm}$ in steps of $3 \mathrm{~mm}$ and then to $45 \mathrm{~mm}$ in steps of $5 \mathrm{~mm}$. As the $\beta$-detection unit was moving away from a sample, it was linearly decreasing up to $30 \%$. Inset is for background of $\beta$-counting channel.

and y-axis is $N_{D} /\left(\varepsilon_{\beta} \times m\right)$ where $m$ is the mass of a considering sample. The observed values of $\beta$-detection efficiency $\varepsilon_{\beta}$ were $0.54 \leq \varepsilon_{\beta} \leq 0.81$. The $N_{D} /\left(\varepsilon_{\beta} \times m\right)$ were varying linearly on the whole counting interval, thus could be linearly extrapolated to $\left(1-\varepsilon_{\beta}\right) / \varepsilon_{\beta}=0$, so $\varepsilon_{\beta}=1$, by the least-squares method for a plot of $N_{D} /\left(\varepsilon_{\beta} \times m\right)$ versus $\left(1-\varepsilon_{\beta}\right) / \varepsilon_{\beta}$. The average of the weighted mean value is $396.3 \pm 1.7 \mathrm{kBq} / \mathrm{g}$ (Table 1 ), at reference date. The uncertainty was calculated from the square root of the summed squares of each uncertainty component as shown in Table 1. The largest uncertainty component was due to the curve fitting. The KRISS certified value is $396.0 \pm 2.0 \mathrm{kBq} / \mathrm{g}$. It is consistent with ours within uncertainty limit.

\section{Selection of the Duration of Dead Time}

Concerning the duration of dead-time, it usually applies
Table 1. The Relative Standard Uncertainty Components

\begin{tabular}{lccc}
\hline \multirow{2}{*}{ Component } & \multicolumn{2}{c}{ Uncertainty (\%) } & \\
\cline { 2 - 3 } & Present & Ref [2] & \\
\hline Counting statistics & 0.05 & 0.05 & $\rho_{\beta}^{1 / 2} / \rho_{\beta}$ \\
Weighting & 0.10 & 0.10 & $\Delta \mathrm{m} / \mathrm{m}$ \\
Dead time & 0.05 & 0.05 & \\
Displacement & 0.04 & 0.04 & \\
Resolving time & 0.02 & 0.02 & \\
Background & 0.08 & 0.57 & $\Delta \mathrm{N}_{B} / \rho_{\beta}$ \\
Extrapolation of efficiency curve & 0.41 & 0.35 & \\
Combined uncertainty & 0.43 & 0.68 & \\
\hline
\end{tabular}

${ }^{a)}$ Quoted from Table 1 of reference [2].

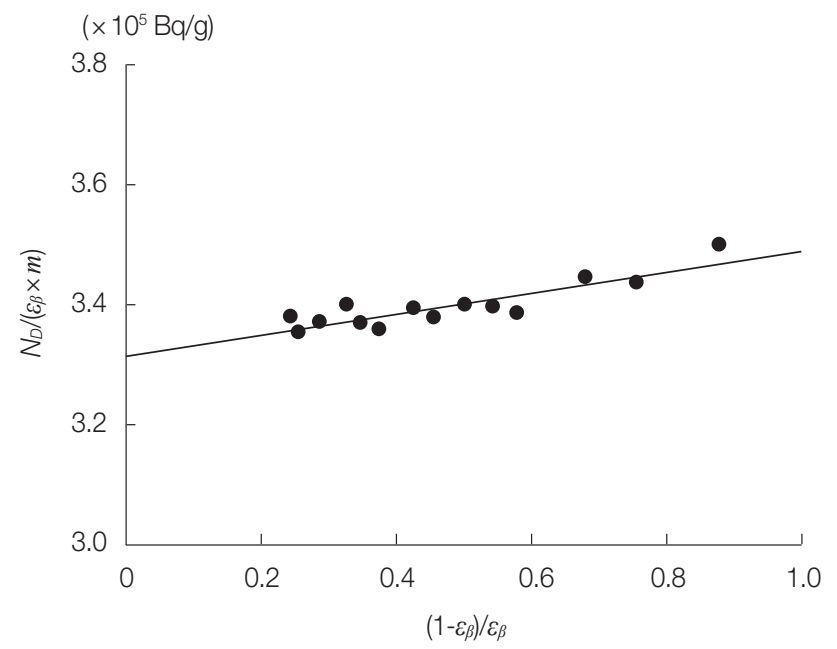

Fig. 4. Efficiency function obtained by means of geometrical efficiency variation method developed first through present work, where $\varepsilon_{\beta}=\rho_{\beta \gamma} / \rho_{\gamma}, N_{D}=\rho_{\beta}$ and $m$ is the mass of a counting sample. It was linearly extrapolated to $\left(1-\varepsilon_{\beta}\right) / \varepsilon_{\beta}=0$ by the least-squares method for a plot of $N_{D} /\left(\varepsilon_{\beta} \times m\right)$ versus $\left(1-\varepsilon_{\beta}\right) / \varepsilon_{\beta}$.

long length of extending type, for example $30 \mu$ s or more, to reject the after-pulses with conventional TDCR method [3-5]. Since we have applied non-extending type for all $\beta$ - and $\gamma$-counting channels, it was necessary to determine the appropriate width of dead-time and also to investigate the effects of after-pulses or other factors on measured values. For this, activity-deviations due to the duration of dead time were observed with non-extendable width varying from $5 \mu$ s to $40 \mu \mathrm{s}$. All measurement cases were performed as described above. To see it more clearly, it has been done with a sample with relatively high counting rate of $6 \times 10^{3} \mathrm{~s}^{-1}$.

The results obtained from each selected width (Fig. 5), were uniformly distributed around the value at $20 \mu$ s. The biggest difference was observed between the data of $5 \mu$ s and of $15 \mu$ s and it was $0.26 \%$ at most. In fact, any notable effects 


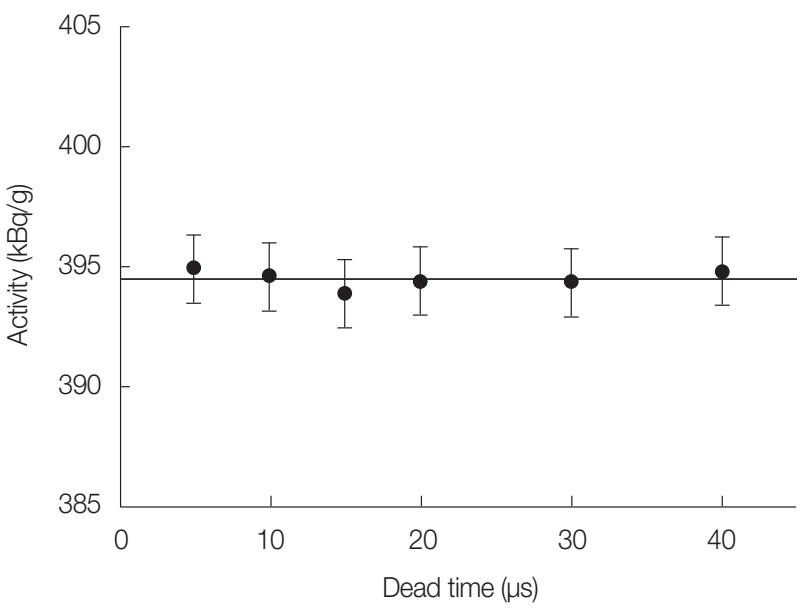

Fig. 5. Plots of the activity determined with each non-extendable dead-time varying from $5 \mu$ s to $40 \mu s$ with a sample of relatively high count rate of $6 \times 10^{3} \mathrm{~s}^{-1}$. All the observed values were distributed uniformly. The dead time of each counting channel was adjusted to be 20 us of non-extending type with uncertainty of $0.05 \%$.

due to after-pulses or other factors on activity determinations could not be found according to our data. So, it was adjusted to be $20 \mu \mathrm{s}$ of non-extending type with a standard uncertainty of $0.05 \%$ for our system.

\section{Conclusion}

We have successfully improved a movable 3PM- $\gamma$ coincidence counting method by using the logical sum of three double coincidences for $\beta$-event. Background due to thermal noises or after-pulses could be reduced to negligible levels. In fact, compared to our earlier work, the measurement accuracy has been much improved.

In addition, there are several positive attributes with liquid scintillation counting such as simple sample preparation, high counting efficiency and the absence of self-absorption. However, to induce efficiency variations with conventional method, either high voltage or SCA adjustment or several quenched material should be prepared. These tasks are not only cumbersome but can also be a source of error. On the other hand, the method developed through this study shows that the efficiency function can be derived by changing the distance between the detector and the sample simply. Not only the measurement conditions are unchanged except the distance but the operation is very easy and fast. The result obtained with the method is well consistent with the KRISS calibrated value.

Consequently, the method developed through this study can be of a suitable technique for activity standardization of $\beta-\gamma$ emitters.

\section{Conflict of Interest}

No potential conflict of interest relevant to this article was reported.

\section{References}

1. Hwang HY, Kwak SI, Lee HY, Lee JM, Lee KB, Park TS. Development of 3-PM liquid scintillation counting system with geometrical efficiency variation. Appl Radiat Isot. 2004;60:469-473.

2. Hwang HY, Sung KS, Lee KB, Lee JM, Park TS. Standardization of radionuclide by $\beta(\mathrm{LS})-\gamma$ coincidence counting using the geometry-efficiency variation method. Appl Radiat Isot. 2006;64: 1119-1123.

3. Pochwalski K, Broda R, Radoszewski T. Standardization of pure beta emitters by liquid-scintillation counting. Int J Rad Appl Instrum A. 1988;39:165-172.

4. Cassette P, Bouchard J. The design of a liquid scintillation counter based on the triple to double coincidence ratio method. Nucl Instrum Methods Phys Res A. 2003;505:72-75.

5. Broda R, Cassette P, Kossert K. Radionuclide metrology using liquid scintillation counting. Metrologia. 2007;44:S36-S52.

6. Bobin Ch, Bouchard J. A $4 \pi(L S) \beta-\gamma$ coincidence system using a TDCR apparatus in the beta-channel. Appl Radiat Isot. 2006;64: 124-130.

7. Johansson LC, Sephton JP. Validation of a new TDCR system at NPL. Appl Radiat Isot. 2010;68:1537-1539.

8. Ziemek T, Jeczmieniowski A, Cacko D, Broda R, Lech E. A new $4 \pi(\mathrm{LS})-\gamma$ coincidence counter at NCBJ RC POLATOM with TDCR detector in the beta channel. Appl Radiat Isot. 2016;109:290-295.

9. Sochorova J, Auerbach P. Activity standardization of ${ }^{134} \mathrm{Cs}$ and ${ }^{137}$ Cs. Appl Radiat Isot. 2014;87:207-210.

10. da Silva CJ, da Cruz PA, Iwahara A, Loureiro JD, Gomes RD, Dos Santos AR, et al. ${ }^{134} \mathrm{Cs}$ activity standardization by $4 \pi \beta(\mathrm{LS})-\gamma(\mathrm{NaI}-$ $\mathrm{Tl})$ anticoincidence counting and submission to international reference system. Appl Radiat Isot. 2018;134:316-320.

11. Be MM, Chiste V, Dulieu C, Browne E, Chechev V, Kuzmenko N, et al. Table of Radionuclides [Internet]. Sevres, France: Bureau International des Poids et Mesures; c2017 [cited 2020 May 31]. Available from: https://www.bipm.org/en/publications/scientific-output/monographie-ri-5.html

12. Cox DR, Isham V. A bivariate point process connected with electronic counters. Proc R Soc Lond A Math Phys Sci. 1977;356:149160.

13. Smith D. Improved correction formulae for coincidence counting. Nucl Instrum Methods. 1978;152:505-519. 